\title{
Cloud discrimination in probability density functions of limb-scattered sunlight measurements
}

\author{
E. N. Normand ${ }^{1}$, J. T. Wiensz ${ }^{2}$, A. E. Bourassa ${ }^{1}$, and D. A. Degenstein ${ }^{1}$ \\ ${ }^{1}$ Institute of Space and Atmospheric Studies, University of Saskatchewan, Canada \\ ${ }^{2}$ SRON Netherlands Institute for Space Research, Utrecht, Netherlands
}

Correspondence to: A. E. Bourassa (adam.bourassa@usask.ca)

Received: 19 April 2013 - Published in Atmos. Meas. Tech. Discuss.: 16 July 2013

Revised: 31 October 2013 - Accepted: 4 November 2013 - Published: 9 December 2013

\begin{abstract}
A technique characterizing the distribution of cirrus cloud-top occurrences from the Optical Spectrograph and Infrared Imaging System (OSIRIS) limb-scattering radiance profiles is presented. The technique involves computing scattering residual profiles by comparing normalized measured radiance and modelled molecular radiance profiles where enhancements in the measured radiance indicate the presence of clouds. Probability density functions of scattering residuals show the distribution is not a continuum measurement; there is a distinction between the cloudy and cloud-free conditions. Observations show high cloud-top occurrences in the upper troposphere and lower stratosphere region above Indonesia and Central America. Results obtained using this technique with OSIRIS measurements are compared to those obtained by Sassen et al. (2008) with Cloud-Aerosol Lidar Pathfinder Satellite Observations (CALIPSO) nadir measurements and to those obtained by Wang et al. (1996) with Stratospheric Aerosol and Gas Experiment (SAGE) II solar occultation measurements.
\end{abstract}

\section{Introduction}

Clouds have pivotal influence on the Earth's hydrological cycle and climate system because they are intricately involved in the dynamical, chemical, and radiative processes within the upper troposphere and lower stratosphere (UTLS) (Chahine, 1992; Liou, 1992; Hobbs, 1993). Cirrus clouds occur at high altitude around the tropopause level and, despite their thin appearance and low optical thickness, they have an important contribution to the radiative balance of the atmosphere (Liou, 1986, 2002). The processes in this region of the atmosphere have become increasingly important for a clear understanding of feedback mechanisms in the climate system.

Detecting and discriminating clouds can be non-trivial. Cloud visibility depends on several factors such as the viewing geometry of the measuring instrument; the relative brightness between the targeted cloud and its background; and the scattering phase function, which characterizes scattering directionality (Sassen et al., 1989). The optical thickness, $\tau$, which is a function of wavelength, is a critical component affecting visibility. Sassen and Cho (1992) categorized cirrus clouds into three groups according to their optical thickness: subvisual clouds with $\tau<0.03$, threshold visible with $\tau \approx 0.03$, and thin cirrus with $\tau>0.03$. Optically thin clouds are below the detection threshold of passive nadirviewing instruments, yet they scatter light sufficiently to bias their trace gas retrievals. By contrast, in limb-scattering or occultation geometries, even very optically thin clouds can produce a measurable effect on the measured brightness profile.

We present a technique for mapping the occurrence frequency and distribution of high-altitude cirrus clouds with satellite measurements of limb-scattered sunlight. When viewed from the Earth's surface with a ground-based zenith lidar system or remotely using nadir sounding, subvisual clouds appear invisible because of their extremely low optical thickness and relative contrast to their background (Sassen et al., 1989). An advantage of the limbscattering remote sensing technique is that subvisual clouds are readily measurable. Limb-scatter measurements have been used previously with other techniques for cloud detection. For example SCIAMACHY (SCanning Imaging 
Absorption SpectroMeter for Atmospheric CHartographY) measurements were used by Eichmann et al. (2010) in a multi-wavelength approach to determine global cloud-top heights, and von Savigny et al. (2004) developed a technique for detection of noctilucent clouds. Limb-emission measurements in the infrared have also been employed for studying clouds. Greenhough et al. (2005) used MIPAS (Michelson Interferometer for Passive Atmospheric Sounding) measurements to retrieve a cloud detection index, and Sembhi et al. (2012) studied MIPAS detection limits for cloud and aerosol particles. We use measurements from the Optical Spectrograph and Infrared Imaging System (OSIRIS), a Canadian satellite instrument that measures atmospheric limb profiles of scattered solar radiation, along with a statistical approach to characterize and discriminate cloud scattering.

A detailed description of the cloud detection technique is presented and the results obtained using the technique are compared to those by Sassen et al. (2008), who used CloudAerosol Lidar Pathfinder Satellite Observations (CALIPSO) nadir measurements and to those by Wang et al. (1996), who used Stratospheric Aerosol and Gas Experiment (SAGE) II solar occultation measurements of cirrus clouds.

\section{Measurements and modelling}

OSIRIS (Llewellyn et al., 2004), a Canadian instrument onboard the Swedish Odin satellite (Murtagh et al., 2002), was designed to measure vertical profiles of atmospheric limb radiance of scattered sunlight from the upper troposphere to the lower mesosphere.

Odin was launched 20 February 2001 into a sunsynchronous orbit with a period of $96 \mathrm{~min}$. The orbital inclination of $98^{\circ}$ from the Equator provides near-global coverage as the corresponding sampled latitude range for nominal on-track instrument pointing is from $82^{\circ} \mathrm{S}$ to $82^{\circ} \mathrm{N}$. The satellite track is near-terminator, implying a dawn-dusk orbit. The entire atmosphere at the tangent point is illuminated when the solar zenith angle is less than $90^{\circ}$. As such, the winter hemisphere is largely in darkness at the local time. Over the course of a year, both the solar zenith angle and the solar scattering angle vary between $60^{\circ}$ and $120^{\circ}$ (Murtagh et al., 2002).

OSIRIS is composed of two optical modules: the optical spectrograph (OS), which is of main interest in this work, and the infrared imager. The OS consists of an optical grating and a CCD detector and measures atmospheric limb radiance between 280 and $810 \mathrm{~nm}$ with a spectral resolution of approximately $1 \mathrm{~nm}$. The OS has a single line of sight, and vertical profiles from roughly 7 to $110 \mathrm{~km}$ in altitude are obtained by nodding the entire spacecraft; this facilitates obtaining observations over a range of tangent altitudes (Murtagh et al., 2002). The OS has a $1 \mathrm{~km}$ vertical and approximately $40 \mathrm{~km}$ horizontal field of view at the tangent point. Successive measurements are separated by roughly $2 \mathrm{~km}$ tangent altitude. It takes nearly $1.5 \mathrm{~min}$ to complete a full vertical scan; thus there are about 60 scans per orbit. Refer to Llewellyn et al. (2004) for a complete description of the instrument.

SASKTRAN (Bourassa et al., 2008) is a spherical geometry radiative transfer model designed to simulate limbscattered solar radiation. It solves the equation of radiative transfer through the method of successive orders for rays travelling within the spherical geometry in order to accurately and efficiently account for the multiple-scattering contribution to the limb radiance. The method of successive orders is used to obtain a solution describing light that has undergone multiple scattering by computing scattering solutions recursively. When modelling the scattering of light by aerosols, SASKTRAN accounts for an altitudedependent cross section and phase function. Absorption by numerous temperature-dependent species is also considered in the model. SASKTRAN is used for operational retrievals of ozone, nitrogen dioxide, and aerosols from the OSIRIS measurements. In this work, SASKTRAN is used to model the radiance from the molecular background in an aerosoland cloud-free atmosphere within a limb geometry. The air number density is taken from European Centre for MediumRange Weather Forecasts (ECMWF) reanalysis interpolated in time and space to the position of the OSIRIS measurement.

\subsection{Single scattering in an optically thin atmosphere}

The radiative transfer equation considering only a singlescattering event of a solar photon in the atmosphere, $I_{1}$, is

$I_{1}\left(\boldsymbol{r}_{0}, \hat{\Omega}\right)=\tilde{I}_{1}\left(s_{1}, \hat{\Omega}\right) e^{-\tau\left(s_{1}, 0\right)}+\int_{s_{1}}^{0} \kappa(s) J_{1}(s, \hat{\Omega}) e^{-\tau(s, 0)} \mathrm{d} s$,

where $\tilde{I}_{1}$ is the radiance from the end of the line of sight; $\boldsymbol{r}_{0}$ is the satellite position; and $s_{1}$ is the position of the singlescattering event along $s$, the axis running along the line of sight. The single-scattering source term, $J_{1}(s, \hat{\Omega})$, is

$J_{1}(s, \hat{\Omega})=\frac{\kappa_{\text {scat }}(s)}{\kappa(s)} F_{0}\left(\hat{\Omega}_{0}\right) e^{-\tau(\operatorname{sun}, s)} \bar{P}\left(s, \hat{\Omega}, \hat{\Omega}_{0}\right)$,

where $\kappa_{\text {scat }}(s)$ is the scattering extinction, $F_{0}\left(\hat{\Omega}_{0}\right)$ is the solar irradiance, $\tau$ (sun, $s$ ) is the optical depth from the Sun to the scattering point, and $\bar{P}\left(s, \hat{\Omega}, \hat{\Omega}_{0}\right)$ is the effective phase function for scattering from the solar direction, $\hat{\Omega}_{0}$, into the propagation direction, $\hat{\Omega}$, and has units per steradian.

In Eq. (1), the exponential $e^{-\tau\left(s_{1}, 0\right)}$ in the first term describes the attenuation of light from the end of the line of sight to the observation point where $s=0$ and the exponential $e^{-\tau(s, 0)}$ in the integral describes the attenuation of light along $s$ to the reference point at $s=0$. The consideration of the single-scattering case implies the solar direction is not aligned with the propagation direction. Therefore, the first term in Eq. (1), which describes radiation emanating from the end of the line of sight, is zero; that is, a limb line of 
sight that ends in deep space. Successive rays in the multiplescatter calculation that strike the ground include this term as ground scatter.

This integral is largely dominated by the tangent point contribution, so for an optically thin atmosphere - that is, when $\tau \ll 1-$ the radiance is

$$
\begin{aligned}
I_{1}\left(\boldsymbol{r}_{\mathbf{0}}, \hat{\Omega}\right) & \approx \kappa\left(s_{\mathrm{T}}\right) J_{1}\left(s_{\mathrm{T}}, \hat{\Omega}\right) \Delta s_{\mathrm{T}}, \\
& \approx \kappa_{\mathrm{scat}}\left(s_{\mathrm{T}}\right) F_{0}\left(\hat{\Omega}_{0}\right) \bar{P}\left(s, \hat{\Omega}, \hat{\Omega}_{0}\right) \Delta s_{\mathrm{T}},
\end{aligned}
$$

where $\Delta s_{\mathrm{T}}$ is the tangent point path length and $\bar{P}\left(s, \hat{\Omega}, \hat{\Omega}_{0}\right)$ is the scattering phase function which describes the probability of scattering in a direction. When a number of particles and molecules contribute to the scattering of light, the radiance is a sum over each scattering contributor. In the case of an atmosphere containing background molecular nitrogen and oxygen as well as particles such as aerosols and clouds, the radiance for an optically thin atmosphere, which is proportional to the number density of the scattering contributors, is

$$
\begin{aligned}
I_{1}\left(\boldsymbol{r}_{\mathbf{0}}, \hat{\Omega}\right) \approx & {\left[n_{\text {scat }_{\mathrm{m}}}\left(s_{\mathrm{T}}\right) \sigma_{\text {scat }_{\mathrm{m}}}\left(s_{\mathrm{T}}\right) P_{\mathrm{m}}\left(s, \hat{\Omega}, \hat{\Omega}_{0}\right)\right.} \\
& \left.+n_{\text {scat }_{\mathrm{p}}}\left(s_{\mathrm{T}}\right) \sigma_{\mathrm{scat}_{\mathrm{p}}}\left(s_{\mathrm{T}}\right) P_{\mathrm{p}}\left(s, \hat{\Omega}, \hat{\Omega}_{0}\right)\right] F_{0}\left(\hat{\Omega}_{0}\right) \Delta s_{\mathrm{T}},
\end{aligned}
$$

where the subscripts $\mathrm{m}$ and $\mathrm{p}$ denote molecular background and particle scattering, respectively.

\section{Cloud detection technique}

\subsection{Scattering residual and probability density functions}

In the limb geometry, the Earth's atmosphere is optically thin down to upper tropospheric tangent altitudes for wavelengths longer than around $700 \mathrm{~nm}$. For this analysis, OSIRIS measurements at $800 \mathrm{~nm}$ were used; this is essentially the longest wavelength measured by OSIRIS that is not contaminated by polarization anomalies caused by the grating. As seen in Eq. (4), the measured radiance, $I_{\text {measured }}$, can be split into two components: that due to the molecular background, $I_{\text {molecular }}$, and due to clouds and aerosols, $I_{\text {particles }}$.

The SASKTRAN model was used to approximate the molecular background radiance within an atmosphere free of aerosols and clouds. By comparing of the measured radiance profiles from OSIRIS to the modelled ones obtained using SASKTRAN, the radiance contribution from clouds and aerosols can be quantified.

The measured radiance and modelled molecular radiance profiles were directly compared after employing a tangent altitude normalization. The reference tangent altitude was chosen nearest $35 \mathrm{~km}$ because it is typically cloud- and aerosolfree at this altitude and because OSIRIS measurements above this altitude start to become contaminated by larger noise due to exponentially falling signal levels. Only scans taken during the descending track of Odin's orbit were considered, which, for a short time period and narrow latitude band, the scattering angle is roughly constant.

In a cloud- and aerosol-free atmosphere, the normalized measured radiance, $\tilde{I}_{\text {measured }}$, and the normalized molecular radiance, $\tilde{I}_{\text {modelled }}$, profiles should essentially agree within the accuracy of the optically thin approximation. The limb radiance is approximately exponential in altitude for an optically thin atmosphere. The discrepancy between the curves within 12 and $35 \mathrm{~km}$ tangent altitudes, shown in the left plot of Fig. 1a, is enhanced scattering due to stratospheric aerosol. Further discrepancies in the measurements indicate the presence of additional aerosol or cloud scattering. We define the difference between the logarithm of the measured and modelled molecular radiance as a scattering residual, $R$, that can be used to characterize scattering enhancements,

$R=\ln \left(\frac{\tilde{I}_{\text {measured }}}{\tilde{I}_{\text {modelled }}}\right)$.

In a cloud-free atmosphere, the residual values in the righthand plot of Fig. 1a hover around zero except in the region where there is a contribution from stratospheric aerosol. Figure $1 \mathrm{~b}$ shows a positive enhancement between 13.7 and $16.1 \mathrm{~km}$ tangent altitudes, suggesting the presence of clouds.

Scattering residual profiles were generated and used to create histograms of scattering residuals. In the absence of clouds the residual values linger around zero, so collectively they form a histogram peak near zero and represent the cloud-free condition. In the event of a cloud, scattering is enhanced so the residual values are much greater and form a second histogram peak. The histogram was normalized by the total number of measurements to obtain a probability density function (PDF). The tangent altitude regions of interest, which are defined by the local tropopause of the scan, were defined to be $1 \mathrm{~km}$ thick and a residual PDF was made for each region. The local tropopause height is defined by the cold point tropopause and is calculated from ECMWF reanalysis at OSIRIS scan points. For a given latitudinal band, these PDFs form a two-dimensional probability density surface shown in Fig. 2. The left maximum range represents the cloud-free condition and the smaller right maximum range represents the cloudy condition. An interesting and useful property of these PDFs is that the distribution in the residual PDF is not a continuum measurement. The separation of the peaks, which is a significant result, provides a clear indication of the ability to distinguish the two conditions.

\subsection{Cloud-free threshold as a function of altitude}

Statistically, integrating a PDF between two limits yields the probability of occurrence of such event within the range of interest. The object here is to separate the cloudy and cloudfree conditions by defining a threshold line that lies between the two distributions. Then, to obtain the probability of locating a cloud within the defined tangent altitude region and 
latitudinal band, the PDF is integrated from the threshold position over the cloudy condition.

Figure 2 shows the cloud-free threshold curve as a function of altitude overtop the probability density surface for scans in the Northern Hemisphere tropical latitudinal band in August 2007 at $800 \mathrm{~nm}$. The distributions corresponding to the cloud-free and cloudy conditions gradually shift to slightly higher residual values as altitude increases due to increasing stratospheric aerosol concentration with altitude. Thus, the threshold distinguishing the two conditions is a curved line as a function of altitude. Although they maintain the same general shape, the maxima cloud-free and cloudy distributions are also different for each latitudinal band due to shifting satellite viewing geometry and any atmospheric variation with latitude. Therefore, each two-dimensional probability density surface requires a unique cloud-free threshold curve. A technique was developed to determine the threshold based on the PDFs.

For each altitude region of interest, the maximum of the cloud-free peak was found and the distance from zero along the residual axis, $\delta_{i}$, was noted, where $i$ denotes the $i$ th altitude region of interest (ROI). The PDF was then normalized by the maximum value of the cloud-free peak and shifted as to align the maximum with zero. This process is shown in Fig. 3a. Once the PDFs from each altitude region were shifted, the PDFs were normalized and averaged to obtain the averaged PDF, which is shown in Fig. $3 b$.

The average cloud-free distribution can be closely approximated by a Gaussian distribution. However, the presence of some residual scattering on the positive side of the peak causes a skew in the distribution. Therefore, the left-hand side of the averaged cloud-free maximum peak was mirrored to obtain a Gaussian-type mirrored shape. The mirrored shape was then fit to a Gaussian.

The variance of the fitted Gaussian, $\sigma^{2}$, was computed such that

$\sigma=\frac{\left|x_{o}-\mu\right|}{\sqrt{2 \ln 2}}$

where $x_{o}$ and $\mu$ are the half-width, half-maximum position and the mean of the fitted Gaussian, respectively. The position $2 \sigma$ was found to be a reliable demarkation of the threshold position between the cloud-free and cloudy conditions. As latitude increases, the distinction between the cloud-free and cloudy distributions becomes blurred, especially near lower tangent altitudes. To determine the cloud-free threshold curve as a function of altitude, the $2 \sigma$ position to the right of the cloud-free peak maximum was determined on the PDFs for each altitude region of interest. That is, the PDFs from each altitude region of interest from Fig. 3 were unshifted by $\delta_{i}$, the distance the original PDF was shifted to zero along the residual axis, and the position $2 \sigma$ to the right of the maximum of the cloud-free peak was marked as the threshold point.
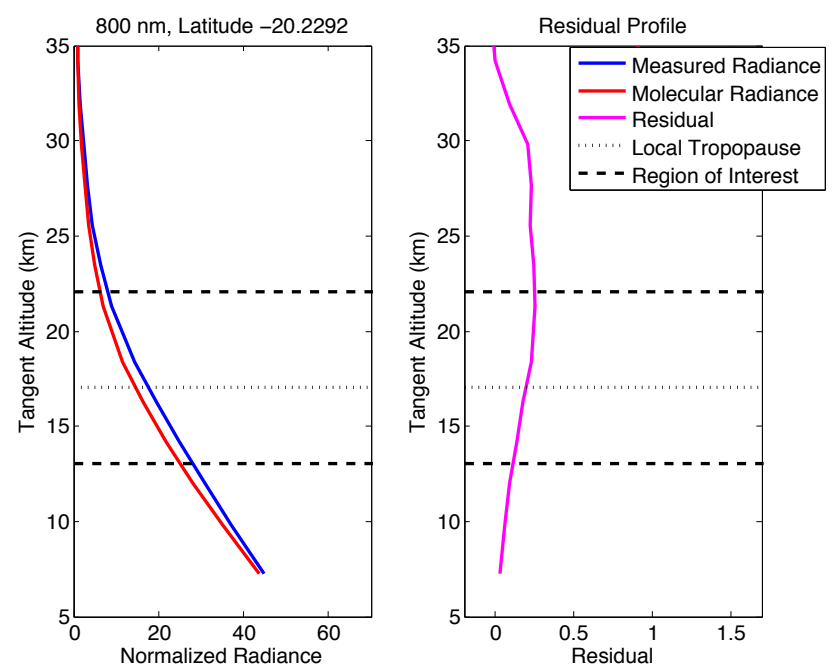

(a) Cloud-free condition
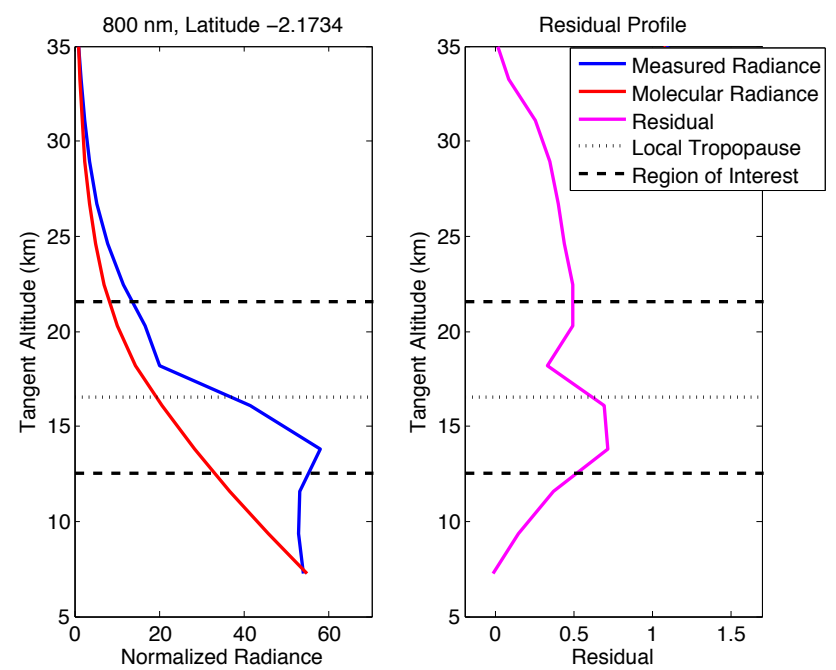

(b) Cloudy condition

Fig. 1. Normalized radiance, density, and residual profiles as a function of tangent altitude at $800 \mathrm{~nm}$ for (a) cloud-free and (b) cloudy conditions. These profiles were measured in May 2007.

The validity of the cloud-free threshold curve is illustrated by computing the residuals, $R$, from simulated OSIRIS measurements through cirrus clouds. This radiative transfer modelling of simulated OSIRIS measurements was done with SASKTRAN by assuming cloud-scattering properties from the in situ database of Baum et al. (2005). The cloud particle number density profile, $n(h)$, is assumed to be Gaussian and is scaled to give a prescribed value of cloud optical thickness, $\tau_{\mathrm{c}}$. The vertical extent of the cloud is defined as the full width at half-maximum (FWHM) of the distribution. Both the use of a single effective particle size and horizontal homogeneity within the cloud layer are assumed. For more details on model configurations, see Wiensz et al. (2013).

Simulated OSIRIS measurements were constructed for 15 August 2007 using the solar geometry for a typical 


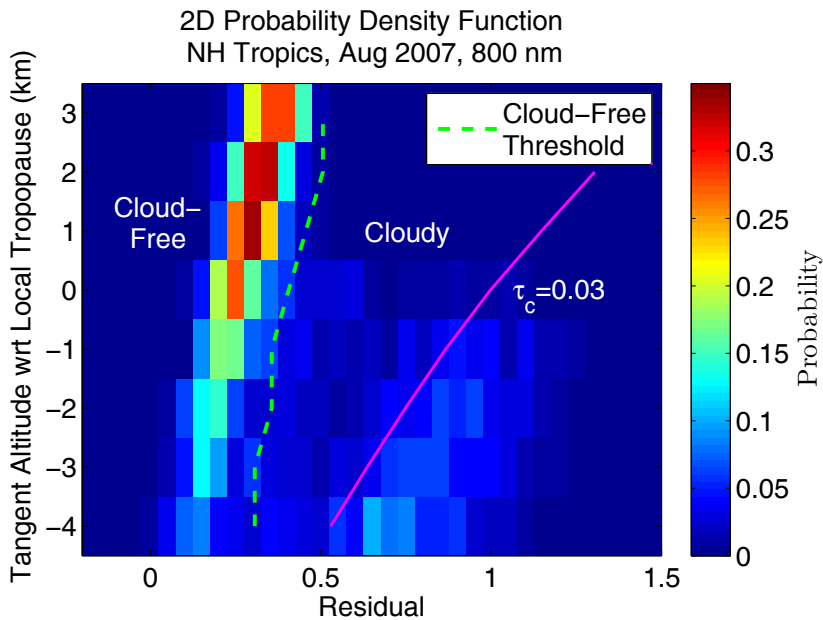

Fig. 2. Two-dimensional residual probability density function (PDF) with the cloud-free threshold curve (dashed green) and corresponding modelled subvisual cirrus curve for cirrus optical thickness $\tau_{\mathrm{c}}=0.03$ (solid magenta) for scans within the Northern Hemisphere tropical latitudinal band in August 2007 at $800 \mathrm{~nm}$. The vertical axis is shown with respect to the local tropopause.

descending-node OSIRIS scan at a latitude of $7^{\circ} \mathrm{N}$. Simulated tangent altitudes were fixed with respect to the local tropopause altitude. To study the perturbation to the values of $R$ from a cirrus cloud at varying altitudes, successive model runs were done with a given cloud (with fixed vertical and optical thickness) as it moved upward through the tangent altitudes. The cloud "bottom" in each case is made to coincide with the line-of-sight tangent altitude to ensure that each line of sight passes directly through the bulk scattering region of the cloud as it is shifted. Residuals were computed from the modelled radiances by Eq. (5). For each set of modelled radiances, which correspond to varying cloud altitude, the maximum residual occurs at the tangent altitude passing through the bulk of the cloud. This value is taken as the residual for the cloud altitude. The curve of computed residual, $R$, as a function of cloud altitude is shown in Fig. 2 for cirrus optical thickness $\tau_{\mathrm{c}}=0.03$, which is the subvisual cirrus detection threshold (Sassen and Cho, 1992). Simulations were done for cloud effective particle size $D_{\mathrm{e}}=40 \mu \mathrm{m}$ and vertical thickness $200 \mathrm{~m}$.

The figure illustrates several key points. First, the cloudfree threshold lies at values of $R$ well below those for subvisual cirrus clouds. This suggests that the threshold indeed forms a demarcation between regions that contain relatively weakly and strongly scattering particles. Second, it is notable that the area of decreased probability between the two "branches" in the figure lies at altitudes and residuals between the threshold curve and the subvisual cirrus curve. This indicates an increased occurrence of thin clouds at values $\tau_{\mathrm{c}} \geq 0.03$ relative to lower $\tau_{\mathrm{c}}$ values.

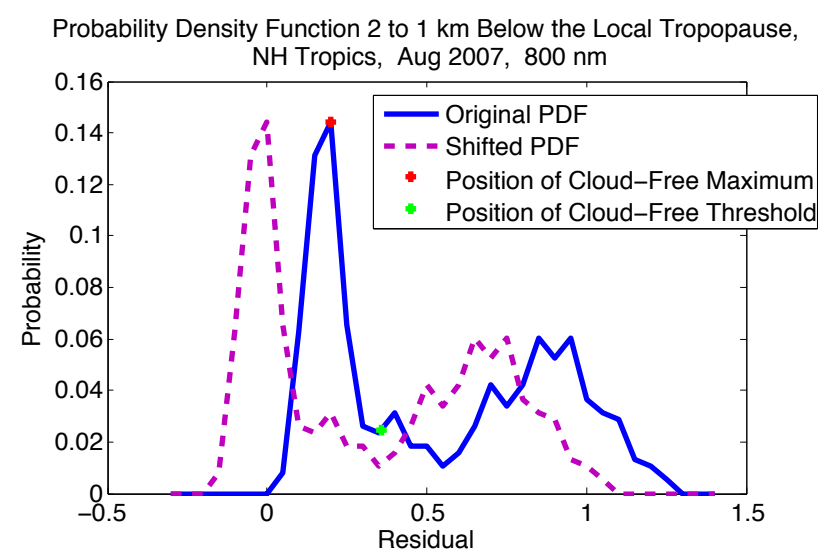

(a)

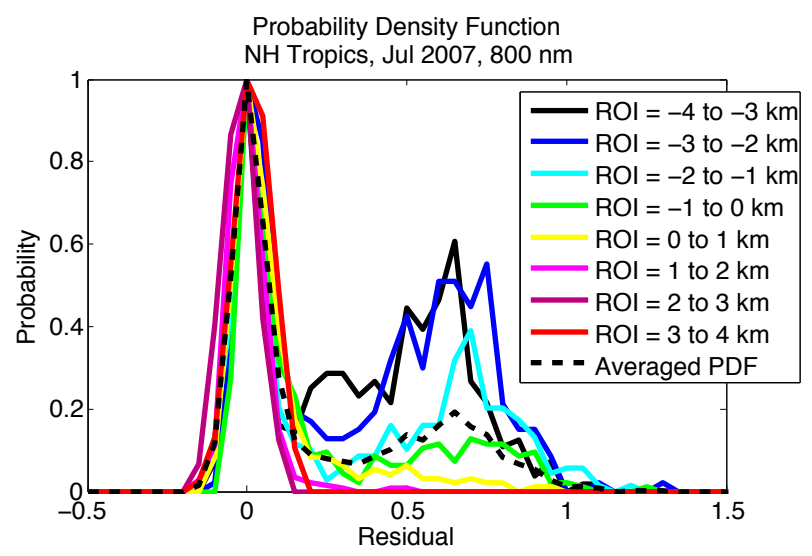

(b)

Fig. 3. (a) PDF for scans within 2 to $1 \mathrm{~km}$ below the tropopause within the Northern Hemisphere tropical latitudinal band in $\mathrm{Au}$ gust 2007 at $800 \mathrm{~nm}$. (b) Normalized and shifted PDFs for various altitude regions of interest (ROI) and averaged PDF for scans within the Northern Hemisphere tropical latitudinal band in July 2007 at $800 \mathrm{~nm}$.

\subsection{Change in solar scattering angle with time}

The solar scattering angle varies over the course of a year due to orbital configurations. Figure 4 shows the monthly average solar scattering angle from June 2006 to June 2007. Roughly, the solar scattering angle follows an approximate cosinusoidal pattern covering a full period over a year, and generally this is the case. However, during the months of October and November 2006, Odin was tilted off the orbital plane so as to acquire measurements poleward of $82^{\circ} \mathrm{S}$. As the solar scattering angle changes with time, the amplitude of the radiance signal measured by OSIRIS also varies with time and causes the cloud-free and cloudy distributions on the PDFs to shift along the residual axis. 


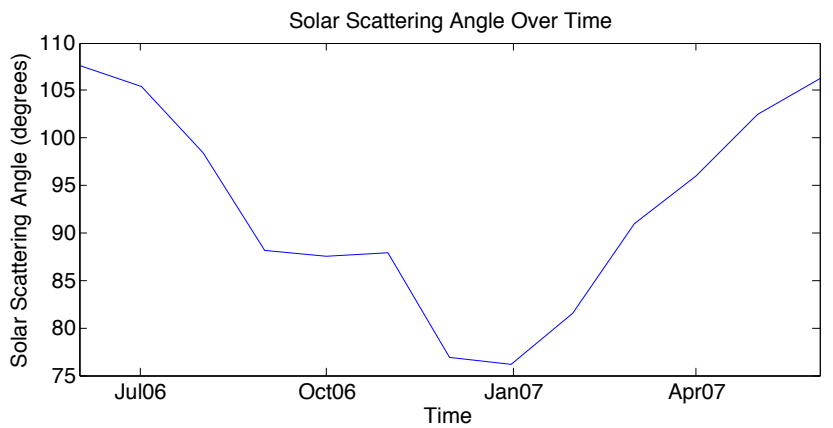

Fig. 4. Solar scattering angle as a function of time from June 2006 to June 2007.

When data spanning a large time period are used to form a single PDF, the distributions are blurred as if there were several time-dependent PDFs overlaid on top of each other. Because the amplitude of the measured signal changes over time due to changing solar scattering angles, it is necessary to separate the data into time-dependent sections as to produce PDFs with little blurring. In this work, data were separated into monthly bins where a two-dimensional PDF, such as that shown in Fig. 2, was made and a unique threshold line was computed for each latitude range and for each month considered.

\subsection{Cloud-top correction}

The OSIRIS scans were organized on a monthly basis into bins according to their latitude and longitude coordinates, where the latitudinal and longitudinal bins were $7.5^{\circ}$ and $20^{\circ}$ wide, respectively. A probability density surface of scattering residuals was created from radiance profiles. A cloud-free threshold curve was determined for each month and latitude band. By integrating from this line over the cloudy maximum range, the probability of locating a cloud for a given altitude range within a latitude-longitude bin on a monthly basis was determined. As neither the vertical thickness nor the horizontal extent of the clouds were taken into account, these probabilities are biased. In order to avoid a counting bias, clouds should only be detected once at the highest detected tangent altitude.

To correct this bias, each scan was checked for residual values that fell in the tangent altitude region of interest beyond the cloud-free threshold curve. The cloud-free threshold curve is a function of altitude; the dashed green line in Fig. 2 separates the cloud-free and cloudy conditions. Thus, the threshold curve was overlaid on the residual profile. The first and highest cloud occurrence beyond the threshold curve was noted and all other residual values below this altitude were disregarded. Therefore, rather than simply detecting the presence of a cloud at a given altitude, the results show the detection of cloud tops; that is, the highest measurement that shows the presence of cloud.

\section{Comparison to CALIPSO}

In an effort to validate and test the cloud detection technique, results using OSIRIS data are compared to those obtained by Sassen et al. (2008), who utilized CALIPSO measurements.

CALIPSO is a joint satellite mission between NASA Langley Research Center and CNES to investigate the impact clouds and aerosols have on the radiative balance of the atmosphere. CALIPSO was launched April 2006 into a circular sun-synchronous polar orbit at about $705 \mathrm{~km}$ altitude. Like Odin, CALIPSO has an orbital inclination of $98^{\circ}$ from the Equator which provides global coverage from $82^{\circ} \mathrm{S}$ to $82^{\circ} \mathrm{N}$. The satellite retraces its track to within $\pm 10 \mathrm{~km}$ every 16 days (Winker et al., 2004, 2007).

Among the three nadir-viewing instruments onboard CALIPSO, Cloud-Aerosol Lidar with Orthogonal Polarization (CALIOP) is a polarization-sensitive lidar and takes measurements of the total attenuated backscatter at 532 and $1064 \mathrm{~nm}$. The lidar profiles contain information on the vertical distribution of clouds and aerosols, the ice-water phase composition of the clouds through the ratio of the signals from two orthogonal polarization channels, and the size distribution of aerosol particles through the wavelength dependence of the backscatter signal (Winker and Pelon, 2003).

Sassen et al. (2008) studied the global distribution of cirrus clouds from CALIPSO measurements from 15 June 2006 to 15 June 2007. A cirrus cloud identification algorithm was developed to assure only cirrus clouds detected by CALIPSO were used in the analysis. Additional constraints were applied to the data to void detections of polar stratospheric clouds (PSCs) and ice clouds occurring at unusually low altitudes. Lastly, in order to distinguish cloud layers, clouds must be separated by a minimum of $1.0 \mathrm{~km}$ in height (Sassen et al., 2008).

Figure 1 of Sassen et al. (2008) shows the global distribution of the average cirrus cloud occurrence frequency as detected by the CALIPSO identification algorithm in terms of $5.0^{\circ}$ longitude and $5.0^{\circ}$ latitude grid boxes. Figure 2 of Sassen et al. (2008) displays the equivalent distribution as altitude versus latitude in $0.2 \mathrm{~km}$ height intervals and $2.5^{\circ}$ latitude bins. For comparison, the analogous average cloudtop occurrence frequency of clouds detected by OSIRIS using the cloud detection technique are shown in Fig. 5a and b, respectively, with $20^{\circ}$ longitude and $7.5^{\circ}$ latitude grid boxes and $2 \mathrm{~km}$ height intervals. The comparisons reveal good agreement especially considering the different viewing geometries of the instruments.

The areas where there is maximum cirrus cloud coverage occur predominantly in the tropical belt at relatively high altitudes. In Fig. 5a, the cirrus cloud occurrence frequency is slightly higher than $60 \%$ over Indonesia and the western part of the Pacific Ocean, and smaller maxima with $\sim 55 \%$ occurrence frequency occur over Central and South America and western-central Africa. These are known regions of intense convective activity: the monsoons in Indonesia, the 


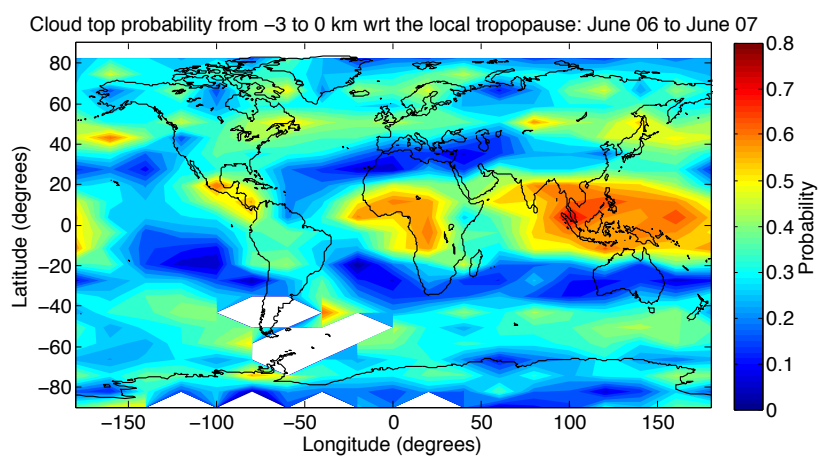

(a) Average cloud top occurrence by OSIRIS

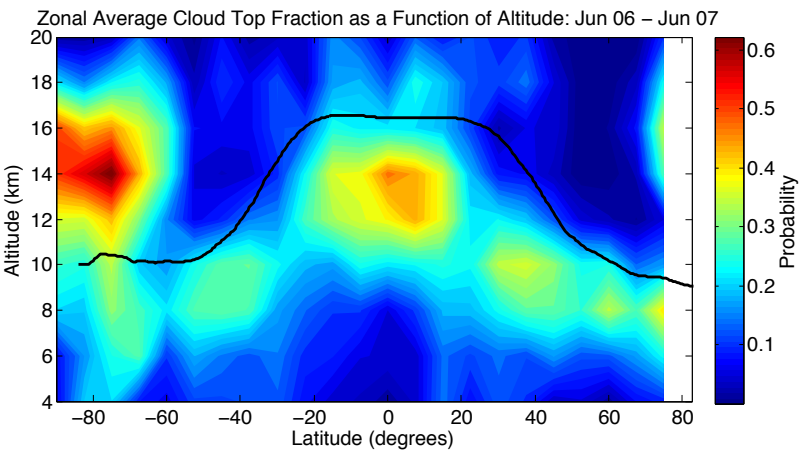

(b) Zonal average cloud top occurrence by OSIRIS

Fig. 5. (a) Average cloud-top occurrence frequency of clouds detected by OSIRIS algorithm in a $3 \mathrm{~km}$ thick layer below the local tropopause between June 2006 and June 2007. (b) Zonal average cloud-top occurrence frequency of clouds detected by OSIRIS algorithm between June 2006 and June 2007.

south-east Asian rainforest, the rainforests in Central America and in the Amazon region, and the Congo Basin rainforest in Africa (Wang et al., 1996; Dessler and Sherwood, 2004; Fu et al., 2007; Sassen et al., 2009). The minima bands along $\pm 30^{\circ}$ latitude correspond to the dry downwelling on the edge of the Hadley cells.

From Fig. 5b, the latitudinal distribution shows maximum cirrus cloud occurrence within the tropical belt between $\pm 15^{\circ}$ latitude at $14 \mathrm{~km}$ altitude, which is coincident with the mean location of the Intertropical Convergence Zone (ITCZ) and the level of maximum convective outflow. There is also about $23 \%$ cloud occurrence in the lower stratosphere within the tropics. These ultra-thin stratospheric clouds are observed by OSIRIS but are not detected by CALIPSO, likely due to differing instrument sensitivities and viewing geometries. Cirrus cloud occurrence generally decreases as the poles are approached except at the South Pole, where the presence of PSCs dominate. PSCs occur well within the stratosphere near $14 \mathrm{~km}$ at high southern latitudes and were removed from the CALIPSO detections. As latitude increases, the cloud-free and cloudy PDF distributions, as in Fig. 2, tend to merge at the lower tangent altitudes, which at these latitudes is closer to the tropopause, and increases the uncertainty in the technique.

\section{Comparison to SAGE II}

The cloud detection technique with OSIRIS limb-scattering measurements is further compared to those obtained by Wang et al. (1996), who used SAGE II occultation measurements as the viewing geometries of these two instruments are similar.

The SAGE II instrument was aboard the Earth Radiation Budget Satellite and its mission was to measure the vertical profiles of ozone, nitrogen dioxide, water vapour, and the aerosol extinction coefficient. The satellite flew in a sunsynchronous orbit with a $90 \mathrm{~min}$ period and a $57^{\circ}$ inclination to allow a latitudinal coverage of approximately $135^{\circ}$ per month. The latitude extremes varied seasonally; however there was no sampling poleward of $55^{\circ}$ during boreal and austral winters (Wang et al., 1996).

SAGE II was a seven-channel radiometer with channels centred at $0.385,0.448,0.453,0.525,0.600,0.940$, and $1.02 \mu \mathrm{m}$. The instrument used the solar occultation technique capturing 15 sunrise events in one day. These were approximately equally separated in longitude and exhibited a slight shift in latitude between consecutive measurements. The instrument's field of view was $0.5 \mathrm{~km}$ vertically and $2.5 \mathrm{~km}$ horizontally at the tangent point (Wang et al., 1996).

Wang et al. (1996) assembled a climatology of cloud occurrence frequency based on six years of SAGE II observations between 1985 and 1990. Subvisual and opaque clouds were distinguished by the measurement upper limit extinction coefficient for aerosols. Using the cirrus cloud classification from Sassen and Cho (1992), clouds with extinction coefficients larger than the measurement limit were marked as opaque clouds because the transmitted signal fell beyond the instrument's sensitivity and the cloud profile was restricted to that altitude (Wang et al., 1996). Furthermore, clouds were distinguished from aerosols through the ratio of the extinction coefficients at two wavelengths, namely at 0.52 and $1.02 \mu \mathrm{m}$. Such a ratio contains information on the particle size.

Fueglistaler et al. (2009) compared the mean cirrus cloud occurrence frequencies for opaque and cirrus clouds derived from CALIPSO and SAGE II instruments in Fig. 9a and b of Fueglistaler et al. (2009), respectively. Although both instruments demonstrate that thinner cirrus clouds occur within the tropical tropopause layer and opaque clouds occur at lower altitudes, the curves illustrating the occurrence frequencies do not agree well. The $\tau<0.03$ thin line in Fig. 9b of Fueglistaler et al. (2009) should be contained within the $\tau<0.1$ thin line in Fig. 9a of Fueglistaler et al. (2009). According to Fueglistaler et al. (2009), the differences between the CALIPSO and SAGE II occurrence frequencies are partly due to the dissimilar viewing geometries of the instruments and to the different optical depth thresholds. Figure 6 demonstrates the cloud-top occurrence frequency from OSIRIS measurements between June 2006 and June 2007. Upon visual inspection, the shapes of the tropical latitudinal 


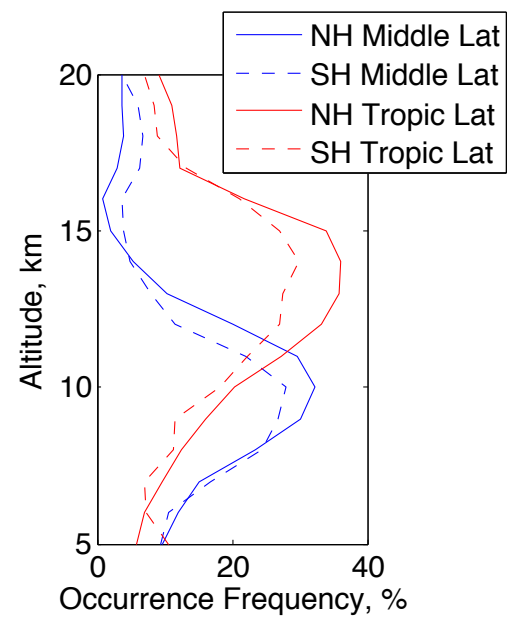

Fig. 6. Mean cloud occurrence frequency versus altitude as measured by OSIRIS from June 2006 to June 2007. Tropical and midlatitudinal bands were defined as $0 \leq \theta<25$ and $25 \leq \theta<55$ in their respective hemispheres.

curves agree nicely with the SAGE II thin cirrus curve. This result is encouraging especially because the two instruments have similar viewing geometries. OSIRIS detected roughly $10 \%$ more cirrus clouds than SAGE II possibly because of a higher limb-viewing sensitivity. This result leads to a confirmation of the theory presented in Fueglistaler et al. (2009).

Plate 1a of Wang et al. (1996) shows the global distribution of cirrus cloud occurrence frequency between 1985 and 1990 as measured by SAGE II. This figure can be compared to Fig. 1 of Sassen et al. (2008) and Fig. 5a. Note, however, that the data used in these figure do not cover the same time period, so differences are to be expected. The comparison between figures must be carried out with care as the figures using CALIPSO and SAGE II data are produced on an absolute altitude scale, while the OSIRIS analysis utilizes an altitude scale relative to the local tropopause. The OSIRIS analysis was carried out in this way to compensate how the tropopause height falls in altitude as the poles are approached. Similarly, the $6 \mathrm{yr}$ average zonal mean occurrence frequency distribution of SAGE II subvisual clouds is shown in Fig. 2a of Wang et al. (1996) and can be compared to Fig. 2 of Sassen et al. (2008) and Fig. 5b. In agreement between these figures and Fig. 6, the maximum cirrus cloud occurrence is between 14 and $15 \mathrm{~km}$ altitude within the tropical latitudinal band. While there are differences between CALIPSO, SAGE II, and OSIRIS measurements, the general positions and magnitudes of the maxima and minima cloud occurrence frequencies occur in relatively close agreement.

To assure adequate sampling, Wang et al. (1996) average six years of SAGE II occultation data together. Since OSIRIS captures enough sampling to form yearly figures, an average figure representing multiple years of data is not necessary and an analysis can be made on a yearly basis. In

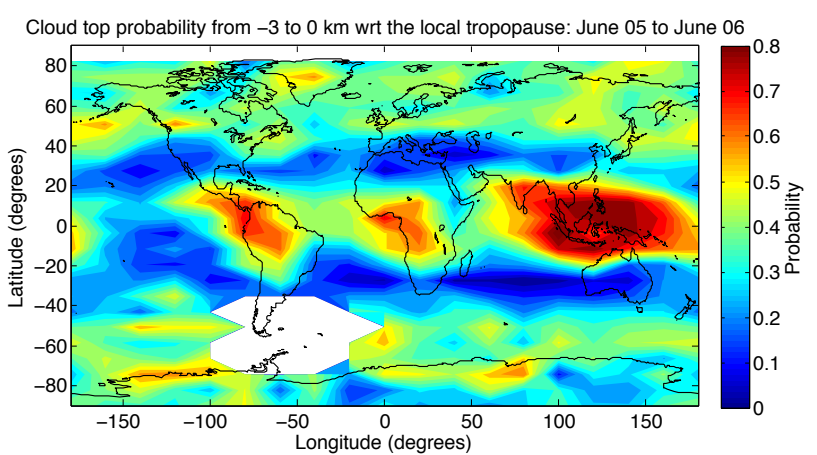

(a)

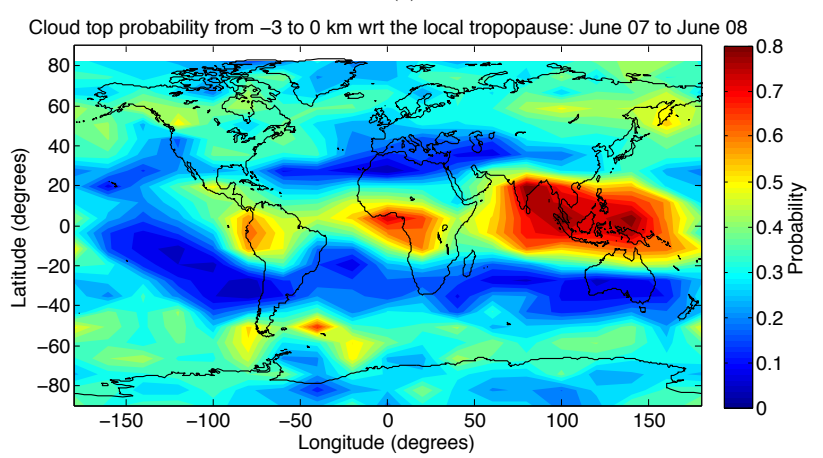

(b)

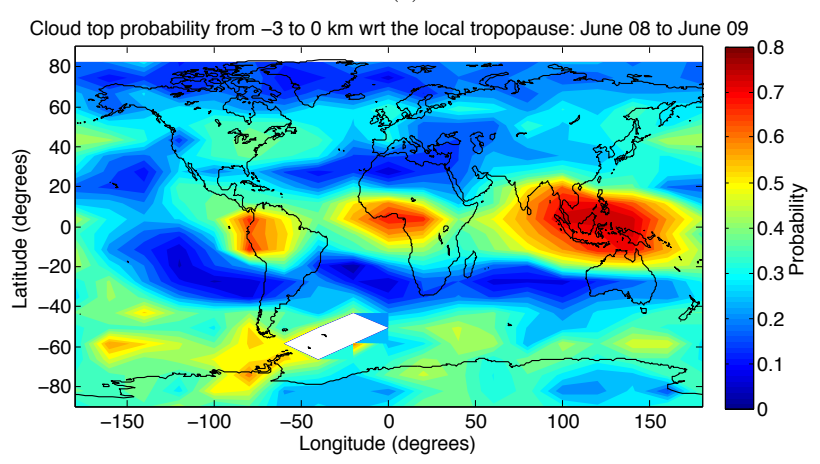

(c)

Fig. 7. Similar to Fig. 5a, yearly average cloud-top occurrence frequency of clouds detected by OSIRIS algorithm in a $3 \mathrm{~km}$ thick layer below the local tropopause.

accompaniment to Fig. 5a, which represents the year 20062007, Fig. 7 shows the yearly average cloud-top occurrence frequency of clouds detected by OSIRIS in a $3 \mathrm{~km}$ layer below the local tropopause for the years 2005-2006, 20072008, and 2008-2009, where each year begins in June. Here again, there are clear maxima cloud occurrences over Indonesia, the Congo rainforest in Africa, and over Central American rain forests, and the Amazon and the downwelling edge of the Hadley cells are neatly identified by the minima bands along $\pm 30^{\circ}$ latitude. 


\section{Conclusions}

OSIRIS employs the limb-scattering technique and measures the radiance of the atmosphere to infer information on the distribution of ozone, nitrogen dioxide, and aerosols within the stratosphere. Although clouds are not measured directly, OSIRIS measurements are used in the development of a highaltitude cloud detection technique.

The efficiency and reliability of the cloud detection technique depends on the residual profile's ability to characterize the occurrence of clouds. The scattering residual is computed as the logarithmic difference between the measured radiance profile at $800 \mathrm{~nm}$ where the atmosphere is optically thin down to tropospheric altitudes and the modelled molecular radiance profile. PDFs are produced from the scattering residual profiles for separate latitudinal bands on a monthly basis. Because the solar scattering angle changes over the course of a year, the variation in the amplitude of the measured radiance signal causes a shift in the PDF distribution along the residual axis. Thus, producing separate time-resolved PDFs prevents blurring distributions.

The PDFs reveal the scattering residual distribution is not a continuum measurement. The ability to distinguish the cloudy and cloud-free conditions is key to the success of the technique. A Gaussian curve is used to model the cloud-free distribution. Threshold residual lines are drawn as a function of altitude two standard deviations to the right of the cloudfree range along the residual axis and delimit the occurrence of clouds. By overlaying these threshold lines onto the residual profiles, the presence of a cloud within the altitude region of interest can be determined. Comparison of the cloud-free threshold with residuals from radiative transfer simulations with known cirrus cloud conditions indicates that the method of threshold selection is reasonable.

A useful application of the cloud detection technique is to produce probability maps showing the distributions of cloudtop occurrence frequencies. Cloud-top maxima were found over Central America and over Indonesia as these correspond to highly convective regions, and minima bands were located along $\pm 30^{\circ}$ latitude on the edge of the Hadley cells.

Fueglistaler et al. (2009) showed profiles of the mean cloud occurrence frequency versus altitude in the tropics as measured by CALIPSO and SAGE II and theorized that the inconsistency between the profiles is a result of the different viewing geometries and optical depth thresholds of the instruments. For comparison, the cloud detection technique was used to plot the cloud-top occurrence frequency versus altitude profiles derived from OSIRIS measurements for tropical and mid-latitude regions. The shape of the tropical profiles compared relatively well with the analogous cirrus cloud profile from SAGE II, which is an encouraging result because SAGE II and OSIRIS have similar viewing geometries.

The applications and use of the cloud detection technique have not been exhausted in this work. Since complex radiative transfer is required to accurately model scattering through optically thick layers of atmosphere or clouds, the cloud detection technique can be used to identify the presence of clouds as well as the cloud-top altitude within the scans. Furthermore, the cloud detection technique can be employed to study the evolution of cloud-top occurrence frequencies and distributions as well as to detect PSCs at high southern latitudes during austral spring.

Acknowledgements. This work was supported by the Natural Sciences and Engineering Research Council (Canada) and the Canadian Space Agency. Odin is a Swedish-led satellite project funded jointly by Sweden (SNSB), Canada (CSA), France (CNES) and Finland (Tekes).

Edited by: A. Kokhanovsky

\section{References}

Baum, B. A., Heymsfield, A. J., Yang, P., and Bedka, S. T.: Bulk Scattering properties for the remote sensing of ice clouds. Part I: Microphysical Data and Models, J. Appl. Meteor., 44, 18851895, 2005.

Bourassa, A. E., Degenstein, D. A., and Llewellyn, E. J.: SASKTRAN: a spherical geometry radiative transfer code for efficient estimation of limb scattered sunlight, J. Quant. Spectrosc. Ra., 109, 52-73, 2008.

Chahine, M. T.: The hydrological cycle and its influence on climate, Nature, 359, 373-380, doi:10.1038/359373a0, 1992.

Dessler, A. E. and Sherwood, S. C.: Effect of convection on the summertime extratropical lower stratosphere, J. Geophys. Res., 109, D23301, doi:10.1029/2004JD005209, 2004.

Eichmann, K.-U., Bovensmann, H., Meringer, M., von Savigny, C., and Kokhanovsky, A.: 38th COSPAR Scientific Assembly, 38, p. 104, 2010.

Fu, Q., Hu, Y., and Yang, Q.: Identifying the top of the tropical tropopause layer from vertical mass flux analysis and CALIPSO lidar cloud observations, Geophys. Res. Lett., 34, L14813, doi:10.1029/2007GL030099, 2007.

Fueglistaler, S., Dessler, A. E., Dunkerton, T. J., Folkins, I., Fu, Q., and Mote, P. W.: Tropical tropopause layer, Rev. Geophys., 47, RG1004, doi:10.1029/2008RG000267, 2009.

Greenhough, J., Remedios, J. J., Sembhi, H., and Kramer, L. J.: Towards cloud detection and cloud frequency distributions from MIPAS infra-red observations, Adv. Space Res., 36, 800-806, doi:10.1016/j.asr.2005.04.096, 2005.

Hobbs, P. V.: Aerosol-Cloud-Climate Interactions, Academic Press, San Diego, Ca., USA, 1993.

Liou, K. N.: Influence of cirrus clouds on weather and climate processes: a global perspective, Mon. Weather Rev., 114, 1167, doi:10.1175/1520-0493(1986)114<1167:IOCCOW>2.0.CO;2, 1986.

Liou, K. N.: Radiation and Cloud Processes in the Atmosphere, Oxford Univ. Press, New York, N. Y., USA, 1992.

Liou, K. N.: An Introduction to Atmospheric Radiation, 2nd Edn., Academic Press, New York, N. Y., USA, 2002. 
Llewellyn, E. J., Lloyd, N. D., Degenstein, D. A., Gattinger, R. L., Petelina, S. V., Bourassa, A. E., Wiensz, J. T., Ivanov, E. V., McDade, I. C., Solheim, I. C., McConnell, J. C., Haley, C. S., von Savigny, C., Sioris, C. E., McLinden, C. A., Griffioen, E., Kaminski, J., Evans, W. F., Puckrin, E., Strong, K., Wehrle, V., Hum, R. H., Kendall, D. J. W., Matsushita, J., Murtagh, D. P., Brohede, S., Stegman, J., Witt, G., Barnes, G., Payne, W. F., Piché, L., Smith, K., Warshaw, G., Deslauniers, D.-L., Marchand, P., Richardson, E. H., King, R. A., Wevers, I., McCreath, W., Kyrölä, E., Oikarinen, L., Leppelmeier, G. W., Auvinen, H., Mégie, G., Hauchecorne, A., Lefèvre, F., de La Nöe, J., Ricaud, P., Frisk, U., Sjoberg, F., von Schéele, F., and Nordh, L.: The OSIRIS instrument on the Odin spacecraft, Can. J. Phys., 82, 411-422, doi:10.1139/P04-005, 2004.

Murtagh, D., Frisk, U., Merino, F., Ridal, M., Jonsson, A., Stegman, J., Witt, G., Eriksson, P., Jiménez, C., Megie, G., de La Noë, J., Ricaud, P., Baron, P., Pardo, J. R., Hauchcorne, A., Llewellyn, E. J., Degenstein, D. A., Gattinger, R. L., Lloyd, N. D., Evans, W. F. J., McDade, I. C., Haley, C. S., Sioris, C., von Savigny, C., Solheim, B. H., McConnell, J. C., Strong, K., Richardson, E. H., Leppelmeier, G. W., Kyrölä, E., Auvinen, H., and Oikarinen, L.: Review: An overview of the Odin atmospheric mission, Can. J. Phys., 80, 309-319, doi:10.1139/P01-157, 2002.

Sassen, K. and Cho, B. S.: Subvisual-thin cirrus lidar dataset for satellite verification and climatological research, J. Appl. Meteorol., 31, 1275-1285, doi:10.1175/15200450(1992)031<1275:STCLDF>2.0.CO;2, 1992.

Sassen, K., Griffin, M. K., and Dodd, G. C.: Optical scattering and microphysical properties of subvisual cirrus clouds, and climatic implications, J. Appl. Meteorol., 28, 91-98, doi:10.1175/15200450(1989)028<0091:OSAMPO>2.0.CO;2, 1989.

Sassen, K., Wang, Z., and Liu, D.: Global distribution of cirrus clouds from CloudSat/Cloud-Aerosol Lidar and Infrared Pathfinder Satellite Observations (CALIPSO) measurements, J. Geophys. Res., 113, D00A12, doi:10.1029/2008JD009972, 2008.
Sassen, K., Wang, Z., and Liu, D.: Cirrus clouds and deep convection in the tropics: Insights from CALIPSO and CloudSat, J. Geophys. Res., 114, D00H06, doi:10.1029/2009JD011916, 2009.

Sembhi, H., Remedios, J., Trent, T., Moore, D. P., Spang, R., Massie, S., and Vernier, J.-P.: MIPAS detection of cloud and aerosol particle occurrence in the UTLS with comparison to HIRDLS and CALIOP, Atmos. Meas. Tech., 5, 2537-2553, doi:10.5194/amt-5-2537-2012, 2012.

von Savigny, C., Kokhanovsky, A., Bovensmann, H., Eichmann, K.U., Kaiser, J., Noël, S., Rozanov, A. V., Skupin, J., and Burrows, J. P.: NLC detection and particle size determination: first results from SCIAMACHY on ENVISAT, Adv. Space Res., 34, 851-856, doi:10.1016/j.asr.2003.05.050, 2004.

Wang, P.-H., Minnis, P. McCormick, M. P., Kent, G. S., and Skeens, K. M.: A 6-year climatology of cloud occurrence frequency from Stratospheric Aerosol and Gas Experiment II observations (1985-1990), J. Geophys. Res., 1012, 29407-29430, doi:10.1029/96JD01780, 1996.

Wiensz, J. T., Degenstein, D. A., Lloyd, N. D., and Bourassa, A. E.: Retrieval of subvisual cirrus cloud optical thickness from limb-scatter measurements, Atmos. Meas. Tech., 6, 105-119, doi:10.5194/amt-6-105-2013, 2013.

Winker, D. M. and Pelon, J.: The CALIPSO Mission, IEEE International, geoscience and remote sensing symposium, 1329-1331, 2003.

Winker, D. M., Hunt, W. H., and Hostetler, C. A.: Status and performance of the CALIOP lidar, Society of Photo-Optical Instrumentation Engineers (SPIE) Conference Series, Vol. 5575, edited by: Singh, U. N., 8-15, doi:10.1117/12.571955, 2004.

Winker, D. M., Hunt, W. H., and McGill, M. J.: Initial performance assessment of CALIOP, Geophys. Res. Lett., 34, L19803, doi:10.1029/2007GL030135, 2007. 